\title{
Clinical Effect Observation of Jieyu Decoction in the Treatment of Generalized Anxiety Disorder
}

\author{
Hanmei Xue ${ }^{1}$, Xiaofang Ren ${ }^{2 *}$ \\ ${ }^{1}$ Shaanxi University of Chinese Medicine, Xianyang 712046, China \\ ${ }^{2}$ The Affiliated Hospital of Shaanxi University of Chinese Medicine, Xi'an 710021, China
}

Funding: Supported by Xi'an Municipal Health Commission Project (SZL201933), Xi'an Hospital of Traditional Chinese Medicine (YJ201922).

\begin{abstract}
[Abstract] Objective: To observe the clinical effect of Jieyu decoction in treating generalized anxiety disorder with stagnation-heat in liver meridian type. Methods: There were 72 cases of generalized anxiety disorders outpatient and inpatient patients, and 36 in the control and treatment group. The control group was given oral Deanxit; the treatment group was given Jieyu decoction granules, and necessary psychological counseling was given to patients in both groups. Changes in traditional Chinese medicine syndrome scoring scale, Hamilton scale (HAMA), dynamic electrocardiogram heart rate variability and other indicators were recorded and compared between the two groups. Results: The total effective rate of the control group was $76.5 \%$, and the total effective rate of the treatment group was $80.0 \%$. The curative effect of the treatment group and the control group was significantly higher than that before treatment $(P<0.05)$, and there was no significant difference in the overall curative effect between the two groups $(P>0.05)$. The HAMA scale score of the two groups was significantly lower than that before treatment $(P<0.05)$, and there was no significant difference in the reduction rate of HAMA score between the two groups $(P>0.05)$. The traditional Chinese medicine syndrome scores of the two groups were significantly decreased compared with that before treatment $(P<0.05)$. The traditional Chinese medicine syndrome scores of the treatment group were significantly different from that of the control group after 8 weeks of treatment $(P<0.05)$. Two groups of heart rate variability in $24 \mathrm{~h}$ period of standard deviation between the average normal $\mathrm{R}-\mathrm{R}$ (SDNN), $24 \mathrm{~h}$ per 5 min normal $\mathrm{R}$ - R period between the standard deviation (SDANN), root mean square of phase difference between adjacent $\mathrm{R}-\mathrm{R}$ (RMSSD) and the number of cardiac $50 \mathrm{~ms}$ accounted for the percentage of the total number of cardiac (PNN50) than before treatment significantly increased $(P<0.05), 8$ weeks after treatment the SDNN, SDANN than the control group no significant difference $(P>0.05)$, RMSSD,PNN50 significantly difference compared with control group $(P<0.05)$. Conclusion: Jieyu decoction has good efficacy and high safety in the treatment of generalized anxiety disorder of the type of liver meridian and heat stagnation, which is suitable for clinical promotion and application.
\end{abstract}

Key words: Generalized anxiety disorder; Stagnation-heat in liver meridian type; Jieyu decoction; Deanxit; Clinical research

Publication date: May, 2021; Publication online: 31 May, 2021

*Corresponding author: Xiaofang Ren, 809991943@qq.com

Generalized anxiety disorder is an independent disease characterized by persistent worry, anxiety and tension ${ }^{[1]}$. Its mental anxiety, physical anxiety, motor anxiety and sleep disorders and other symptoms affect the work and life of patients in many ways, serious cases can lead to damage to the social function of patients ${ }^{[2]}$. The study shows that the 12 -month prevalence rate of GAD in China is $0.8 \%$, and the lifetime prevalence rate is $1.2 \%{ }^{[2]}$. During the epidemic, the incidence of adult public anxiety in Panzhihua City, Sichuan Province was as high as $39.4 \%{ }^{[3]}$. Therefore, we urgently 
need effective intervention through drugs and psychotherapy to help patients improve their quality of life in many aspects, such as social psychology, life satisfaction, work efficiency and so on.

At present, the pathogenesis of GAD is not clear, including endocrine, neurotransmitter, immune injury and other aspects ${ }^{[4]}$. Among them, drugs targeting neurotransmitter receptors such as 5-hydroxytryptamine (5-HT) and norepinephrine (NE) are used as the main treatment methods in clinic. These drugs have many adverse reactions and obvious withdrawal reactions at the same time ${ }^{[5]}$. Although there are cognitive behavioral therapy(CBT), Morita therapy and other psychotherapy as auxiliary, but poor compliance, long duration, high economic cost and other problems still make the overall therapeutic effect of GAD is not good ${ }^{[6]}$. A lot of data show that traditional Chinese medicine treatment of GAD is more targeted, and the compatibility of rational prescriptions and medicines is flexible, which greatly avoids the serious adverse reactions of chemical drugs. The mechanism of traditional Chinese medicine in the treatment of GAD has been constantly discovered in the research and exploration of many scholars, which provides more data support for its clinical treatment, and its clinical application is getting wider and wider ${ }^{[7]}$.

\section{Data and methods}

\subsection{General data}

Included in 72 outpatients and inpatients who met the diagnostic criteria of traditional Chinese and western medicine of GAD from January 2020 to January 2021, and were randomly divided into control group and treatment group. The hospital's ethics committee fully understood and approved the study.

\subsection{Diagnostic criteria}

Western medicine diagnostic criteria refer to "Chinese Classification and Diagnostic criteria of Mental Disorders" 3rd edition (CCMD-3) GAD diagnostic criteria; TCM diagnostic criteria refer to "anxiety disorders, guidelines for diagnosis and treatment of common diseases in traditional Chinese medicine" (formulated by the Chinese Society of traditional Chinese Medicine in 2008) ${ }^{[8,9]}$.

\subsection{Patients}

Patients with (HAMA)score $>14$, but $<29$ were included, and those with anxiety attacks and severe psychotic symptoms secondary to other mental or somatic diseases were excluded,and those with poor compliance or taking other antipsychotic drugs during the study period were excluded ${ }^{[10]}$.

\section{Methods}

\subsection{Therapeutic methods}

Control group: Deanxit (haloperidoxine tablets, Hainan Huineng Pharmaceutical Co., Ltd., H20143390, specification: each tablet contains $0.5 \mathrm{mg}$ haloperixine +10 mg melitracine), 1 tablet each time, 1 tablet in the morning and 1 tablet in the noon. Treatment group: Jieyu decoction granule (composed of $15 \mathrm{~g}$ of albizzia bark, $10 \mathrm{~g}$ of Radix Ophiopogonis, $12 \mathrm{~g}$ of Radix Paeoniae Alba, $10 \mathrm{~g}$ of turmeric, $10 \mathrm{~g}$ of bergamot, $6 \mathrm{~g}$ of Rhizoma nardostachyos, $15 \mathrm{~g}$ of Rubia officinalis, $20 \mathrm{~g}$ of tuber fleeceflower stem), 1 dose a day, washed with warm water, and taken in the morning and evening. The course of treatment was 8 weeks. During the study, patients in both groups were not treated with other antipsychotic drugs, and were given necessary psychological counseling at the same time.

\subsection{Clinical efficacy}

The clinical efficacy was judged by the reduction rate of $(\mathrm{T})$ of HAMA scale before and after treatment as the main index of curative effect. Recovery: HAMA score reduction rate $>$ $75 \%$; obvious effective: $50 \% \leq$ HAMA score reduction rate $\leq 75 \%$; effective: $25 \% \leq$ HAMA score reduction rate $<50 \%$ : invalid: HAMA score reduction rate $<25 \%$. Total effective rate $(\%)=$ (number of cured cases + number of markedly effective cases + number of effective cases-shedding) / total number of cases $\times 100 \%$.

\subsection{Statistical analysis methods}

The data are processed by SPSS 22.0 statistical software, and the measurement data are expressed by ${ }^{-} \pm \mathrm{s}$. The measurement data were first tested for normality, and the rank sum test was used for non-normal distribution; paired sample t-test was used for comparison within the measurement data group consistent with normal distribution, and independent sample t-test was used for inter-group comparison. Rank sum test and nonparametric test were used for grade data, and chi-square test was used for counting data. The two-sided test was used in the hypothesis test, and the difference was statistically significant $(\mathrm{P}<$ $0.05)$.

\section{Results}

\subsection{Comparison of general information}


See Table 1. In the course of the study, 3 cases of moving, work and loss of follow-up were included in the control group $(n=34)$ and the treatment group $(n=35)$. There was no significant difference in the general data between the two groups $(\mathrm{P}>0.05)$.

Table 1. General data of two groups of patients

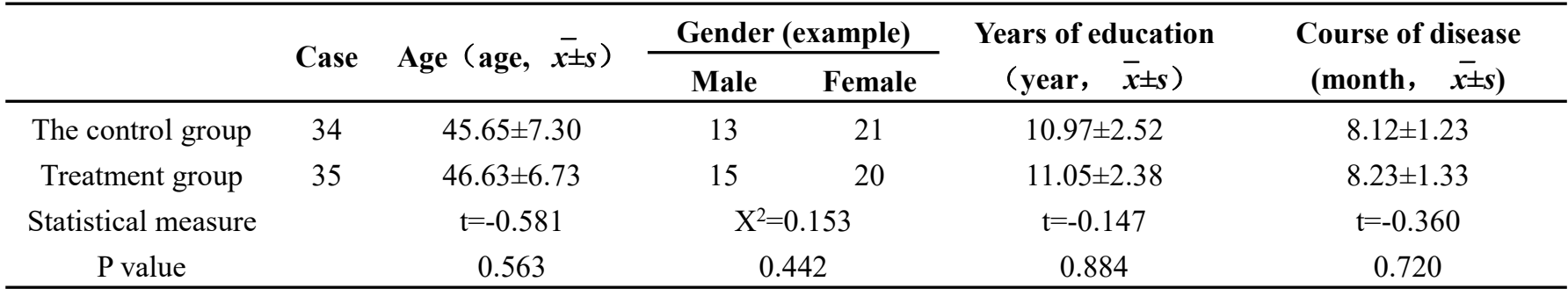

Note: Compared with the control group, $\mathrm{P}>0.05$.

3.2 The comparison of HAMA scale scores between in Table 2. the two groups before and after treatment is shown

Table 2. Comparison of HAMA scores between the two groups before and after treatment ( $\bar{x} \pm s)$

\begin{tabular}{cccc} 
Group & Before treatment & \multicolumn{2}{c}{ After treatment } \\
\cline { 3 - 4 } & & 4 weeks & 8 weeks \\
\hline The control group $(\mathrm{n}=34)$ & $22.06 \pm 4.11$ & $15.82 \pm 3.97^{*}$ & $10.26 \pm 1.83^{*}$ \\
Treatment group $(\mathrm{n}=35)$ & $21.71 \pm 4.05^{\triangle}$ & $14.97 \pm 3.67^{* \Delta}$ & $9.80 \pm 2.00^{* \triangle}$ \\
\hline
\end{tabular}

Note: Compared with this group before treatment, ${ }^{*} \mathrm{P}<0.05$; compared with the control group, ${ }^{\triangle} \mathrm{P}>0.05$.

\subsection{The comparison of the scores of TCM syndrome scale is shown in Table 3 .}

Table 3. Scores of TCM syndrome scale before and after treatment in two groups $(\bar{x} \pm s)$

\begin{tabular}{cccc}
\hline \multirow{2}{*}{ Group } & Before & \multicolumn{2}{c}{ After treatment } \\
\cline { 3 - 4 } & treatment & 4 weeks & 8 weeks \\
\hline The control group $(\mathrm{n}=34)$ & $12.74 \pm 2.63$ & $7.74 \pm 2.63^{*}$ & $5.73 \pm 2.63^{*}$ \\
Treatment group $(\mathrm{n}=35)$ & $13.49 \pm 3.02^{\triangle}$ & $6.60 \pm 3.08^{*}$ & $3.66 \pm 2.44^{*} \triangle \triangle$ \\
\hline
\end{tabular}

Note: compared with this group before treatment, ${ }^{*} \mathrm{P}<0.05$; compared with the control group at the same time, ${ }^{\triangleright} \mathrm{P}>0.05$; compared with the control group at the same time, ${ }^{\Delta \triangle} \mathrm{P}<0.05$.

\subsection{The observation of curative effects before and after treatment is shown in Table 4.}

Table 4. Comparison of therapeutic effects between the two groups [cases (\%)]

\begin{tabular}{|c|c|c|c|c|c|c|c|}
\hline Group & Recovery & $\begin{array}{l}\text { Obvious } \\
\text { effective }\end{array}$ & Effective & Invalid & $\begin{array}{c}\text { Total effective } \\
\text { Rate (\%) }\end{array}$ & $Z$ value & P value \\
\hline $\begin{array}{l}\text { The control group } \\
\qquad(\mathrm{n}=34)\end{array}$ & $3(8.8 \%)$ & $15(44.1 \%)$ & $8(23.5 \%)$ & $8(23.5 \%)$ & $76.5 \%$ & -0.333 & 0.739 \\
\hline $\begin{array}{l}\text { Treatment group } \\
\qquad(\mathrm{n}=35)\end{array}$ & $3(8.6 \%)$ & $17(48.6 \%)$ & $8(22.9 \%)$ & $7(20.0 \%)$ & $80.0 \%$ & & \\
\hline
\end{tabular}

Note: By rank sum test, $\mathrm{P}>0.05$

3.5 Comparison of TESS scale scores and safety between the two groups

As shown in table 5.As evaluated by TESS scale, the minor adverse reactions in both groups were alleviated after proper treatment. 
Table 5. TESS scale scores of the two groups after treatment $(\overline{\mathrm{x}} \pm \mathrm{s})$

\begin{tabular}{cccc}
\hline Group & Adverse reaction score (score, $\overline{\mathbf{x}} \pm$ s) & Statistical measure & P value \\
\hline The control group $(\mathrm{n}=34)$ & $0.65 \pm 0.92$ & $\mathrm{Z}=-2.618$ & 0.009 \\
Treatment group $(\mathrm{n}=35)$ & $0.23 \pm 0.69$ & & \\
\hline
\end{tabular}

Note: By rank sum test, $\mathrm{P}>00.05$

\subsection{The comparison of heart rate variability Table 6.} between the two groups after treatment is shown in

Table 6. Analysis and comparison of heart rate variability between the two groups after treatment $(\overline{\mathrm{x}} \pm \mathrm{s})$

\begin{tabular}{cccccc}
\hline Group & Time & SDNN ( ms) & SDANN ( ms) & RMSSD (ms) & PNN50 ( \%) \\
\hline The control & Before treatment & $92.76 \pm 12.34$ & $94.79 \pm 12.36$ & $30.26 \pm 3.78$ & $9.68 \pm 2.73$ \\
group & After 4 weeks of treatment & $123 \pm 10.27^{*}$ & $120.03 \pm 10.45^{*}$ & $40.00 \pm 4.16^{*}$ & $13.35 \pm 2.87^{*}$ \\
$(\mathrm{n}=34)$ & After 8 weeks of treatment & $127.97 \pm 10.92^{*}$ & $129.29 \pm 10.68^{*}$ & $48.00 \pm 4.16^{*}$ & $18.59 \pm 2.80^{*}$ \\
Treatment & Before the treatment & $89.46 \pm 15.48$ & $90.23 \pm 15.35$ & $32.43 \pm 3.45$ & $8.97 \pm 2.70$ \\
group & After 4 weeks of treatment & $125.37 \pm 9.83^{* \triangle}$ & $123.20 \pm 9.52^{* \triangle}$ & $41.12 \pm 3.24^{* \triangle}$ & $14.00 \pm 2.97^{* \triangle}$ \\
$(\mathrm{n}=35)$ & After 8 weeks of treatment & $129.54 \pm 10.01^{* \triangle}$ & $131.09 \pm 9.36^{* \triangle}$ & $53.37 \pm 2.97^{* \triangle \Delta}$ & $20.26 \pm 2.95^{* \triangle \Delta}$ \\
\hline
\end{tabular}

Note: Compared with this group before treatment, ${ }^{*} \mathrm{P}<0.05$; Compared with the control group, ${ }^{\triangle} \mathrm{P}>0.05$; Compared with the control group, ${ }^{\triangle \triangle} \mathrm{P}<0.05$

\section{Discussion}

GAD's main symptoms are fear of unknown goals or excessive worry about specific goals, as Danxi Heart method said, "personal diseases are often born in depression", so it is classified as "depression syndrome". The internal injury of emotion is the main cause of GAD, and the deficiency of body and the deficiency of visceral qi are the internal factors, as stated in "the origin of miscellaneous diseases, rhinoceros candle and depression". The mixed syndrome of deficiency and excess is often seen in the course of GAD over a long period of time, and the location of the disease is mainly in the liver. The liver is the master of catharsis, it is most comfortable to be in a state of patency, and the depression of liver qi will damage the viscera. As the words of doctors in Liuzhou said, "the disease of seven emotions must start from the liver." Chief physician Ren Xiaofang summarized through clinical diagnosis and treatment that the pathogenesis of GAD is mainly qi-fire depression, fire depression and forcing yin, and the treatment should be soothing the liver and relieving depression, nourishing yin and softening the liver.

Jieyu decoction is a self-made prescription by Professor Yang Zhen. The original prescription was used to treat the "stagnant heat phase fire" stage of hepatitis B. Chief physician Ren Xiaofang, based on the principle of treating different diseases with the same treatment, used it to treat GAD liver Meridian stagnation-heat syndrome. The prescription consists of albizzia bark, Ophiopogon japonicus, Radix Paeoniae Alba, turmeric, bergamot, rhizoma nardostachyos, rubia officinalis, tuber fleeceflower stem.

Albizzia bark, tuber fleeceflower stem for the king medicine, albizzia skin into the heart, liver and lung meridian, have the effect of relieving depression and calming the mind; tuber fleeceflower stem into the heart and liver meridian, nourish the heart and calm the mind, ventilation and blood flow. Turmeric and Radix Paeoniae Alba are courtier medicine, qi depression for a long time, blood stasis, turmeric can help albizzia bark soothing the liver and relieving depression, and has the function of regulating qi, cooling blood and removing blood stasis; Radix Paeoniae Alba nourishes blood and camp, collects yin and calms the liver, nourishes the liver and relieves palpitation. Bergamot, rhizoma nardostachyos, Rubia officinalis and Ophiopogon japonicus are all adjuvants to see the disease of the liver. Bergamot enters the liver, spleen and lung meridian, which can soothe the liver and regulate the spleen, rhizoma nardostachyos enters the spleen and stomach meridian, and can regulate qi and awaken the spleen, which can help soothe the liver and regulate qi, awaken the spleen and stomach; rubia officinalis clear away heat and cool blood, and cool blood without leaving blood 
stasis; Rubia officinalis enters the heart, lung and stomach meridian, which has the effect of nourishing yin, giving birth to fluid, clearing the heart and removing annoyance.

All kinds of medicines are in harmony, playing the effect of soothing the liver and relieving depression, nourishing yin and softening the liver, truly regulating qi without breaking qi, cooling blood without leaving blood stasis, clearing heat without damaging the stomach, tonifying without weariness. Physiological characteristics of using Yang based on liver body Yin, soothing the liver and clearing heat, regulating the liver and nourishing the liver, the liver qi reaches, the visceral qi is filled gradually, and the disease recovers itself.

To sum up, Jieyu decoction in the treatment of GAD with stagnation of liver meridian has the same efficacy as the chemical drug Deanxit, which can significantly improve the symptoms of patients, have a positive impact on some indexes of heart rate variability, and has high safety, so it is worth popularizing and applying in clinic.

\section{Reference}

[1] van der Heiden Colin, Methorst Gerda, Muris Peter et al. Generalized anxiety disorder: clinical presentation, diagnostic features, and guidelines for clinical practice [J]. J Clin Psychol, 2011, 67: 58-73.

[2] Ma X, Xiang YT, Cai ZJ, et al. Generalized anxiety disorder in China: prevalence, sociodemographic correlates, comorbidity and sui-cide attempts [J]. Perspectives in Psychiatric Care, 2009, 45(2): 119-127.
[3] Zhang J, Li Q, Zhang WJ, et al. The anxiety, coping style, social support and correlation of adult public during COVID-19 's period [J]. Chinese Journal of Health Psychology, 2020, 28 (12): 1817-1821.

[4] Xu BY. Research progress on the pathogenesis of generalized anxiety disorder [J]. Sichuan Mental Health, 2012. 25 (03): 188-191.

[5] Shen XH, Shen ZX. Progress in the treatment of generalized anxiety disorder [J]. Medicine and Philosophy (B), 2013 Magi 34 (02): 15-19.

[6] Jiang XJ, Hao FY, Chen NX, et al. Group cognitive behavioral therapy for chronic insomnia $[\mathrm{J}]$. Chinese Clinical Neuroscience, 2017, 25 (01): 112-118.

[7] Zhang RC, Li HN, Liu SW, et al. Research progress on the mechanism of traditional Chinese medicine therapy in the treatment of generalized anxiety disorder [J]. Chinese Journal of traditional Chinese Medicine, 2017 Magi 35 (12): 3015-3018.

[8] Psychiatric Branch of Chinese Medical Association. Classification and diagnostic criteria of mental disorders in China. Jinan: Shandong Science and Technology Publishing House, 2001:53.

[9] Chinese Society of traditional Chinese Medicine. Guidelines for diagnosis and treatment of common diseases in traditional Chinese medicine. Beijing: China traditional Chinese Medicine Publishing House, 2008 rig 294-296.

[10] Hamilton M. The assessment of anxiety states by rating [J]. British Journal of Medical Psychology, 1959: 50-55. 